\title{
Comparative analysis of the effect of two chlorhexidine mouthrinses on plaque accumulation and gingival bleeding
}

\section{Carlos Alfredo Franco Neto(a) Clarissa Cavalcanti Fatturi Parolo(b) Cassiano Kuchenbecker Rösing(c) Marisa Maltz(d)}

(a) Docent; (b)Docent, MS; (d) PhD, Professor - Department of Preventive and Social Dentistry, School of Dentistry, Federal University of Rio Grande do Sul.

(c) $\mathrm{PhD}$, Professor, Department of Conservative Dentistry, School of Dentistry, Federal University of Rio Grande do Sul.
Corresponding author:

Marisa Maltz

Universidade Federal do Rio Grande do Sul

Faculdade de Odontologia

Departamento de Odontologia

Preventiva e Social

Rua Ramiro Barcelos, 2492, Santana

Porto Alegre - RS - Brazil

CEP: 90035-003

E-mail:mmaltz@ufrgs.br

\begin{abstract}
The aim of the present study was to evaluate the effect of two chlorhexidine rinsing solutions $(0.12 \%$ and $0.2 \%)$ on plaque and gingival bleeding. Ten dental students participated in this double-blind, cross-over study, rinsing twice a day, for one minute, with each one of the tested solutions for fourteen days. A wash-out period of one week between treatments was observed. In order to assess gingival bleeding, the van der Weijden et al. ${ }^{1}$ (1994) index was used. The plaque indexes used were those of Quigley, Hein ${ }^{2}$ (1962) and Silness, Löe ${ }^{3}$ (1964). In the pre-experimental period, subjects received oral hygiene instructions and dental prophylaxis. The results revealed no significant differences between both concentrations in relation to plaque and gingival bleeding. Mean values ( \pm standard deviation) of the Quigley \& Hein index were $0.25 \pm 0.16$ for the $0.12 \%$ solution and $0.23 \pm 0.26$ for the $0.2 \%$ solution $(\mathrm{p}=0.4838)$. Mean values ( \pm standard deviation) of the Silness-Löe index were $0.12 \pm 0.10$ for the $0.12 \%$ solution and $0.11 \pm 0.11$ for the $0.2 \%$ solution $(\mathrm{p}=0.7592)$. The bleeding index mean values at the end of the study were not different for both concentrations with mean values ( \pm standard deviation) of $14.93 \% \pm 6.68 \%$ and $13.95 \pm 9.24 \%$ for the $0.12 \%$ and $0.2 \%$ solutions, respectively. Although an increase in gingival bleeding was observed, both concentrations were able to control dental plaque.
\end{abstract}

Descriptors: Chlorhexidine; Dental plaque; Products with antimicrobial action; Gingivitis. 


\section{Introduction}

The most prevalent infectious oral diseases in humans, caries and periodontal diseases, are associated with dental plaque. The removal of bacterial biofilm is a decisive component in the prevention and treatment of these diseases. The use of mechanical agents is a simple and cost-effective method that has been demonstrated to be efficient in gingivitis control. ${ }^{4}$ The effectiveness of this method, however, is influenced by the individual's manual ability and motivation. Because of the difficulty to ensure adequate removal of plaque by mechanical means, there is a great interest in the use of antimicrobial agents to replace or to be adjuncts to the mechanical approaches. Chlorhexidine ( $\mathrm{CHX})$ is one of the most effective antimicrobial agents for plaque control. ${ }^{5-10}$ It is retained in the oral cavity and is progressively desorbed in bacteriostatic concentrations 8 hours after rinsing. ${ }^{11}$ When a low dose of CHX is used, the cellular transport of the bacterial cell is damaged with the creation of pores in the cellular membrane..$^{12}$ In higher concentration, the solution penetrates the bacterial cell and leads to microorganism destruction.

In Europe, a 0.2\% CHX solution was developed and became the standard international concentration. ${ }^{6}$ A lower concentration of $\mathrm{CHX}(0.12 \%)$ has been tested in animals and human populations and has also demonstrated clinical benefits., ${ }^{7,13}$ Although CHX is an effective antimicrobial agent in both concentrations, comparison of the two existing formulations is still necessary. The aim of the present study was to assess the efficacy of two concentrations of chlorhexidine solutions $(0.12 \%$ and $0.20 \%$ ) on plaque development and gingival bleeding control during 14 days.

\section{Material and Methods}

This study involved a randomized controlled clinical trial with a cross-over double-blind design. Ten dental students ( 7 male and 3 female) aged 2025 years were included in the study.

To be included in the study, the volunteers should have at least 24 teeth, not have prosthetic or orthodontic appliances, no previous or present history of periodontitis (the subjects had no site with PPD $>3 \mathrm{~mm}$ and had no site with PAL $>2 \mathrm{~mm}$ ) or any systemic condition that could negatively influence oral health, not have been under antimicrobial treatment locally or systemically in the 90 days previous to the experiment.

Two regimes of chlorhexidine rinsing were prescribed: (a) $15 \mathrm{ml}$ of a $0.12 \%$ chlorhexidine solution, which corresponds to $18 \mathrm{mg}$ of chlorhexidine (Hibitane Dental ${ }^{\circledR}$, ICI Pharmaceuticals PLC, Macclesfield, UK) twice daily; (b) $10 \mathrm{ml}$ of a $0.20 \%$ chlorhexidine solution, equivalent to $20 \mathrm{mg}$ of chlorhexidine (Hibitane Dental ${ }^{\circledR}$, ICI Pharmaceuticals PLC, Macclesfield, UK) twice daily.

Two weeks prior to baseline, a thorough clinical examination was performed in order to identify sites with plaque (determined by visible plaque index $)^{15}$ and gingival bleeding (Gingival Bleeding Index). ${ }^{1}$ The volunteers that had plaque and extrinsic pigmentation were submitted to professional mechanical tooth cleaning with the use of abrasives, rubber cups and brushes. The volunteers with gingivitis received oral hygiene instruction. Calculus was removed by meticulous scaling carried out with manual instruments.

In the beginning of each experimental period, the Gingival Bleeding Index ${ }^{1}$ was assessed. After this, plaque disclosure and prophylaxis were performed. At this moment, the first rinsing was given to the volunteers, who received a $500 \mathrm{ml}$ coded plastic bottle and a corresponding dose dispenser. Half of the volunteers received the $0.12 \% \mathrm{CHX}$ solution and the other half received the $0.2 \% \mathrm{CHX}$ solution. No mechanical plaque control was performed during the experiment.

At day 14 after baseline in each experimental period, subjects were examined using the Quigley \& Hein Plaque Index ${ }^{2}$ followed by the Silness \& Löe Plaque Index ${ }^{3}$ and the Gingival Bleeding Index, according to van der Weijden et al. ${ }^{1}$ (1994). For the Quigley \& Hein Plaque Index, ${ }^{2}$ a photo-activated plaque disclosure system was utilized (Plaque test - Ivoclar Vivadent, Schaan, Liechtenstein), thus not disturbing the following Silness and Löe Plaque assessment.

All the clinical parameters were assessed by one trained experienced examiner under standard dental office and light source conditions.

After the first experimental period, a 1-week 
wash-out interval was given, when the volunteers performed standard mechanical plaque control. Following washout, each volunteer received the opposite regime.

At day 21, following the wash-out period, the volunteers were clinically examined in the same way as at Baseline. At day 35, final examination, similar to that performed at day 14 , took place.

For each Plaque Index, mean values $( \pm$ standard deviation) were calculated at day 14 and 35 (and merged according to the CHX regimen) and statistically analysed by the Wilcoxon rank sign test.

Mean values ( \pm standard deviation) of Gingival Bleeding scores at baseline and at day 14 and 35 (merged according to CHX regimen) were calculated and analysed by the Wilcoxon rank sign test.

The relation between each one of the plaque indices and the Gingival bleeding index was determined by linear regression. The alpha level for all the analyses in this study was set at $5 \%$. The data for this trial was used in order to calculate the power of the study. The primary outcome considered was the Silness \& Löe Plaque Index. ${ }^{3}$ Taking into consideration the study design and the variability of the results, a clinically relevant difference of 0.10 and $\alpha$ error of $0.05, \beta$ error of 0.24 were expected. Thus, the power of this study was $76 \%$.

\section{Results}

Table 1 shows the results concerning plaque accumulation. Fourteen days after plaque accumulation, no statistically significant differences were observed between both chlorhexidine concentration regimes. For the Silness \& Löe Plaque Index, mean values were $0.12( \pm 0.10)$ and $0.11( \pm 0.11)$ and for the Quigley \& Hein Index, mean values were 0.25 $( \pm 0.16)$ and $0.23( \pm 0.26)$ for the $0.12 \%$ and $0.20 \%$ regimes, respectively.

The results concerning the Gingival Bleeding Index are demonstrated in Table 2. At the beginning, both groups displayed around 3\% of gingival bleeding. After 14 days, the group that rinsed with $0.12 \%$ chlorhexidine had a percentage of $14.93( \pm 6.68)$ and the $0.20 \%$ group had $13.95 \%$ ( \pm 9.24$)$. These values were not statistically different. It should be noted that some individuals had up to $30 \%$ of bleeding sites.

The relation between both plaque index systems and the Gingival Bleeding Index is shown in Graphs 1 and 2. For the Quigley \& Hein index, the linear regression coefficients were $\mathrm{R}^{2}=0.77$ and $\mathrm{R}^{2}=0.22$ for the $0.12 \%$ and $0.20 \%$ groups respectively (Graph 1).

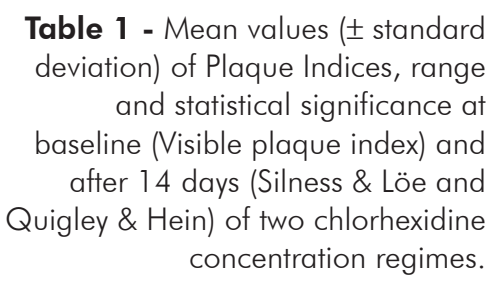

Table 2 - Gingival Bleeding (GB) and Visible plaque (VP) Index (mean \pm standard deviation) and range at baseline and after 14 days of two chlorhexidine concentration regimes.

\begin{tabular}{|c|c|c|c|c|}
\hline Index & $\begin{array}{l}\text { Chlorhexidine } \\
\text { Concentration }\end{array}$ & $\begin{array}{c}\text { Mean } \pm \text { Standard } \\
\text { Deviation }\end{array}$ & Range & $\begin{array}{l}\text { Statistical } \\
\text { significance }\end{array}$ \\
\hline \multirow{2}{*}{ Visible Plaque } & $0.12 \%$ & $5.00 \pm 0.05 \%$ & $0.00-17 \%$ & \multirow{2}{*}{$\mathrm{ns}^{* a}$} \\
\hline & $0.20 \%$ & $6.00 \pm 0.07 \%$ & $0.00-18 \%$ & \\
\hline \multirow{2}{*}{$\begin{array}{l}\text { Silness \& Löe (Silness, } \\
\text { Löe, }^{3} \text { 1964) }\end{array}$} & $0.12 \%$ & $0.12 \pm 0.10$ & $0.00-0.35$ & \multirow{2}{*}{$n s^{* b}$} \\
\hline & $0.20 \%$ & $0.11 \pm 0.11$ & $0.00-0.28$ & \\
\hline \multirow{2}{*}{$\begin{array}{l}\text { Quigley \& Hein } \\
\text { (Quigley, Hein,2 1962) }\end{array}$} & $0.12 \%$ & $0.25 \pm 0.16$ & $0.04-0.46$ & \multirow{2}{*}{$n s^{* c}$} \\
\hline & $0.20 \%$ & $0.23 \pm 0.26$ & $0.00-0.67$ & \\
\hline
\end{tabular}

$n s^{*}$ : Non-significant difference. (Wilcoxon: $\mathrm{ns}^{* a} \mathrm{p}=0.7504 ; n \mathrm{~s}^{* \mathrm{~b}} \mathrm{p}=0.7592 ; n \mathrm{~s}^{* \mathrm{c}} \mathrm{p}=0.4838$ ).

\begin{tabular}{|c|c|c|c|c|}
\hline Period & $\begin{array}{l}\text { Chlorhexidine } \\
\text { Concentration }\end{array}$ & $\begin{array}{l}\text { Mean } \pm \text { Standard } \\
\text { Deviation }\end{array}$ & Range & $\begin{array}{l}\text { Statistical } \\
\text { significance }\end{array}$ \\
\hline \multirow{2}{*}{ Baseline } & $0.12 \%$ & $3.56 \pm 3.60 \%$ & $0.00-10.40 \%$ & \multirow{2}{*}{$\mathrm{ns}^{* \mathrm{a}}$} \\
\hline & $0.20 \%$ & $3.43 \pm 3.43 \%$ & $0.00-10.70 \%$ & \\
\hline \multirow{2}{*}{ Day 14} & $0.12 \%$ & $14.93 \pm 6.68 \%$ & $3.70-27.10 \%$ & \multirow{2}{*}{$n s^{* b}$} \\
\hline & $0.20 \%$ & $13.95 \pm 9.24 \%$ & $1.80-32.10 \%$ & \\
\hline
\end{tabular}

$n s^{*}$ : Non-significant difference (Wilcoxon: $\mathrm{ns}^{* a} \mathrm{p}=1.000 ; n \mathrm{~s}^{* b} \mathrm{p}=0.6784$ ). 

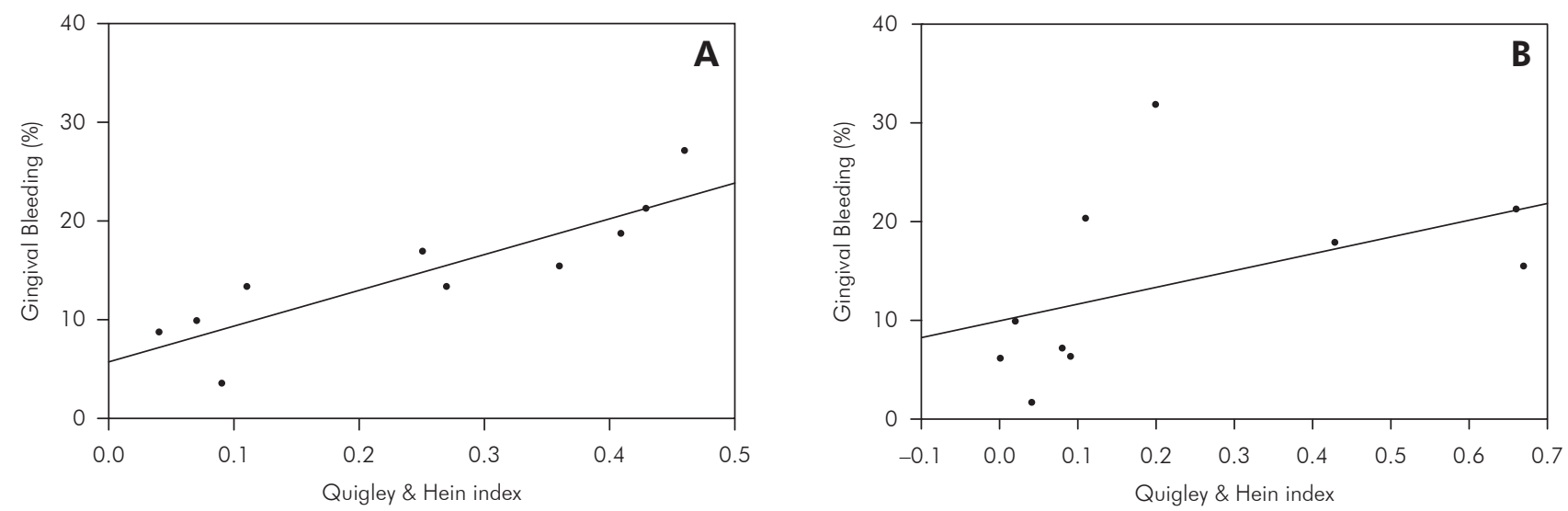

Graph 1 - Linear regression analysis between Gingival Bleeding and Quigley \& Hein index for the 0.12\% (A) and 0.20\% (B) groups.
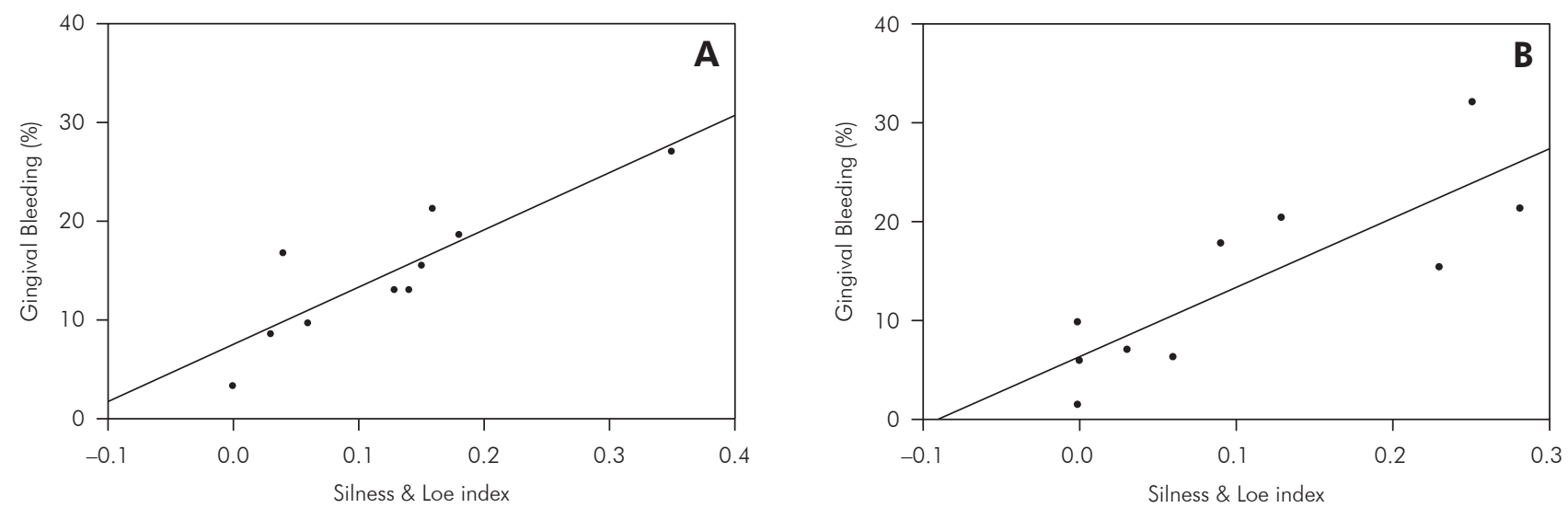

Graph 2 - Linear regression analysis between Gingival Bleeding and Silness \& Löe index for the 0.12\% (A) and 0.20\% (B) groups.

When the Silness \& Löe index was compared with the Gingival Bleeding Index, linear regression coefficients were $\mathrm{R}^{2}=0.75$ and $\mathrm{R}^{2}=0.68$ for the $0.12 \%$ and $0.20 \%$ chlorhexidine concentrations, respectively (Graph 2).

The results showed the relation between the Silness \& Löe index and the Gingival Bleeding Index in both concentrations. For the Quigley \& Hein index, a relation between plaque index and the Gingival Bleeding Index was observed for the $0.12 \%$ concentration but not for the $0.2 \%$ concentration.

\section{Discussion}

The present study evaluated clinically two chlorhexidine concentration regimes as substitutes for mechanical plaque control. A randomized, cross-over, double blind design was chosen in order to generate the best possible evidence. Calibration was not quantified prior to or during the experiment since the clinical parameters evaluated are indicators of the moment, so it is not possible for them to be tested in these terms. Thus, a single trained examiner was responsible for assessments.

The study population comprised dental students, which is an interesting group for this kind of study, ${ }^{9,16}$ taking into consideration the fact that they have lower levels of gingival inflammation and can be easily controlled in terms of compliance. Baseline data indicate this and facilitate plaque accumulation experimental designs.

In terms of plaque accumulation, two studies did not find differences between two chlorhexi- 
dine concentrations. Lang et al. ${ }^{14}$ (1982) observed mean Silness \& Löe Plaque Indices of 1.24 and 1.29 following 6 months of use of $0.20 \%$ and $0.10 \%$ chlorhexidine rinses. When the Quigley \& Hein Index is concerned, Segreto et al. ${ }^{17}$ (1986) demonstrated mean indices of 1.01 for the $0.12 \%$ solution and 1.14 for the $0.2 \%$ solution after 3 months. The difference in terms of plaque accumulation from these studies and the present may be due to the different timing of the experimental settings.

On the other hand, Jenkins et al. ${ }^{18}$ (1989), with an experimental design similar to ours, demonstrated less plaque accumulation when the higher concentration was tested. Ernst et al. ${ }^{19}$ (1998) also did not demonstrate statistically significant differences between $0.1 \%$ and $0.2 \%$ chlorhexidine rinses as adjuncts to mechanical plaque control in a larger sample of 130 subjects. The same result was obtained by Asari et al. ${ }^{20}$ (1996) when these two chlorhexidine concentrations were used as subgingival irrigators.

A point that should not be overlooked is the sample size of the present study. Studies with similar aims have been published with sample sizes similar to that of this study. ${ }^{16,21}$

In our study, no significant differences were observed in terms of gingival bleeding after 14 days. However, gingivitis was observed in $14.93 \%$ and $13.95 \%$ of sites in users of the $0.12 \%$ and $0.20 \%$ chlorhexidine solutions, demonstrating an establishment of inflammation. Jenkins et al. ${ }^{18}$ (1989) also observed some degree of gingival bleeding after controlled-force probing $18.86 \%$ and $10.95 \%$ for the $0.10 \%$ chlorhexidine solution and $6.0 \%$ and $6.29 \%$ for the $0.12 \%$ solution after 12 and 19 days, respectively). The study by Siegrist et al. ${ }^{22}$ (1986) also showed an increase in the Gingival Index from day 7 to $21(6.2 \%$ to $13 \%)$.

The subjects with a higher plaque index at base-

\section{References}

1. van der Weijden GA, Timmerman MF, Nijboer A, Reijerse E, Van der Velden U. Comparison of different approaches to assess bleeding on probing as indicators of gingivitis. J Clin Periodontol. 1994;21(9):589-94. line were the ones with the larger amount of bleeding sites. The relation observed between plaque indices and gingival inflammation confirms this fact, previously demonstrated by Siegrist et al. ${ }^{22}$ (1986) and Brecx et al. ${ }^{23}$ (1992).

A bacterial succession during the 14-day period could explain the results, leading to inflammation even with low plaque scores. Also, it has been postulated that chemical agents would be superior to mechanical means especially in difficult-to-reach areas, but this is not totally supported by the results of this study, since baseline bleeding scores (obtained by mechanical means solely) were lower than after 14 days of chemical plaque control.

The results of this study may contribute to clinical practice in two ways. First, demonstrating that mechanical plaque control is still the gold standard for patients that have the capacity of performing it, since some plaque accumulation and gingival inflammation occurred after 14 days of chemical plaque control. Second, that lower concentrations of chlorhexidine should be prescribed, diminishing adverse effects, since higher concentrations do not seem to generate lower plaque and gingivitis scores. However, it should be noted that the amounts of rinsing solution should be observed (as in the present study) in order to guarantee similar chlorhexidine amounts in the oral cavity.

\section{Conclusions}

No differences were observed in terms of antiplaque efficacy between the $0.12 \%$ and $0.20 \%$ chlorhexidine rinsing solutions. Chlorhexidine rinsing keeps plaque levels low, but allows some degree of gingival inflammation after 14 days.

\section{Acknowledgments}

We thank the volunteers for their dedication.

2. Quigley GA, Hein JW. Comparative cleansing efficiency of manual and power brushing. J Am Dent Assoc. 1962;65:26-9.

3. Silness J, Löe H. Periodontal Disease in Pregnancy II. Correlation between Oral Hygiene and Periodontal Condition. Acta Odontol Scand. 1964;22(1):121-35. 
4. Beltrami M, Bickel M, Baehni PC. The effect of supragingival plaque control on the composition of the subgingival microflora in human periodontitis. J Clin Periodontol. 1987;14(3):1614.

5. Bral M, Brownstein CN. Antimicrobial agents in the prevention and treatment of periodontal diseases. Dent Clin North Am. 1988;32(2):217-41.

6. Hase JC, Attstrom R, Edwardsson S, Kelty E, Kisch J. 6month use of $0.2 \%$ delmopinol hydrochloride in comparison with $0.2 \%$ chlorhexidine digluconate and placebo. (I). Effect on plaque formation and gingivitis. J Clin Periodontol. 1998;25(9):746-53.

7. Keijser JA, Verkade H, Timmerman MF, van der Weijden FA. Comparison of 2 commercially available chlorhexidine mouthrinses. J Periodontol. 2003;74(2):214-8.

8. Lang NP, Brecx MC. Chlorhexidine digluconate - an agent for chemical plaque control and prevention of gingival inflammation. J Periodontal Res. 1986;21(16):74-89.

9. Lorenz K, Bruhn G, Heumann C, Netuschil L, Brecx M, Hoffmann T. Effect of two new chlorhexidine mouthrinses on the development of dental plaque, gingivitis, and discolouration. A randomized, investigator-blind, placebo-controlled, 3-week experimental gingivitis study. J Clin Periodontol. 2006;33(8):561-7

10. van Strydonck DA, Demoor P, Timmerman MF, van der Velden $\mathrm{U}$, van der Weijden GA. The anti-plaque efficacy of a chlorhexidine mouthrinse used in combination with toothbrushing with dentifrice. J Clin Periodontol. 2004;31(8):691-5.

11. Rolla G, Löe H, Schiott CR. Retention of chlorhexidine in the human oral cavity. Arch Oral Biol. 1971;16(9):1109-16.

12. Rye RM, Wiseman D. Effect of chlorhexidine upon ${ }^{32} \mathrm{P}$ release and cell viability in Escherichia coli. J Pharm Pharmacol. 1966;18(S):114-8.

13. Bay LM. Effect of toothbrushing with different concentrations of chlorhexidine on the development of dental plaque and gingivitis. J Dent Res. 1978;57(2):181-5.
14. Lang NP, Hotz P, Graf H, Geering AH, Saxer UP, Sturzenberger OP et al. Effects of supervised chlorhexidine mouthrinses in children. A longitudinal clinical trial. J Periodontal Res. 1982;17(1):101-11.

15. Ainamo J, Bay I. Problems and proposals for recording gingivitis and plaque. Int Dent J. 1975;25(4):229-35.

16. Quirynen M, Avontroodt P, Peeters W, Pauwels M, Coucke W, van Steenberghe D. Effect of different chlorhexidine formulations in mouthrinses on de novo plaque formation. J Clin Periodontol. 2001;28(12):1127-36.

17. Segreto VA, Collins EM, Beiswanger BB, de la Rosa M, Issacs RL, Lang NR et al. A comparison of mouthrinses containing two concentrations of chlorhexidine. J Periodontal Res. 1986;21(16):23-32.

18. Jenkins S, Addy M, Newcombe R. Comparison of two commercially available chlorhexidine mouthrinses: II. Effects on plaque reformation, gingivitis, and tooth staining. Clin Prev Dent. 1989;11(6):12-6.

19. Ernst CP, Prockl K, Willershausen B. The effectiveness and side effects of $0.1 \%$ and $0.2 \%$ chlorhexidine mouthrinses: a clinical study. Quintessence Int. 1998;29(7):443-8.

20. Asari AM, Newman HN, Wilson M, Bulman JS. 0.1\%/0.2\% commercial chlorhexidine solutions as subgingival irrigants in chronic periodontitis. J Clin Periodontol. 1996;23(4):320-5.

21. Sekino S, Ramberg P, Uzel NG, Socransky S, Lindhe J. Effect of various chlorhexidine regimens on salivary bacteria and de novo plaque formation. J Clin Periodontol. 2003;30(10):91925.

22. Siegrist BE, Gusberti FA, Brecx MC, Weber HP, Lang NP. Efficacy of supervised rinsing with chlorhexidine digluconate in comparison to phenolic plant alkaloid compounds. J Periodontal Res. 1986;21(16):60-73.

23. Brecx M, Brownstone BE, MacDonald L, Gelskey S, Cheang M. Efficacy of Listerine, Meridol and chlorhexidine mouthrinses as supplements to regular tooth cleaning measures. J Clin Periodontol. 1992;19(3):202-7. 